\title{
Estrategias de aprendizaje en estudiantes con discapacidad en aulas de educación básica de la Amazonía Peruana
}

\author{
Learning strategies for students with disabilities in basic education classrooms in the Peruvian amazon
}

Estratégias de aprendizagem em alunos com deficiência em salas de aula da educação básica na Amazônia peruana

\section{ARTÍCULO GENERAL}

\author{
Juan Rafael Juarez Diaz \\ https://orcid.org/0000-0002-8113-6932 \\ jrjuarezd@unsm.edu.pe \\ Universidad Nacional de San Martin, San Martín - Perú
}

Recibido 07 de Junio 2021 | Arbitrado y aceptado 25 de Agosto 2021 | Publicado en 04 Diciembre 2021

\section{RESUMEN}

En el proceso de inclusión educativa, se ha intentado diversas formas para aprovechar las condiciones en las que se desarrolla la educación básica, que permita que el estudiante con discapacidad pueda aprender con calidad. Es producto de un estudio no experimental descriptivo propositiva, siendo la muestra de 183 docentes. Se encontró que los docentes de las instituciones regulares consideran deficiente el uso de técnicas básicas para el desarrollo social del niño incluido, escaso uso de las inteligencias múltiples y la participación activa. Los docentes del centro especial, consideran que no se ha puesto importancia en la gestión y los valores de los docentes inclusivos ante el proceso educativo, como la planificación, la identificación de nuevas oportunidades para conseguir agrupaciones heterogéneas, desarrollo social y la resolución de problemas y el uso de instrumentos como el plan de orientación individual y la evaluación psicopedagógica. Ante esto, se diseñó estrategias metodológicas fundamentado en la teoría de la complejidad y las inteligencias múltiples que demuestra su efectividad, tanto en su concepción teórica como en la práctica, demostrando efectividad en el logro de aprendizaje de estudiantes incluidos.

\section{Palabras clave}

Método de enseñanza, deficiencia mental, incapacidad, estudiante y metodología.

\section{ABSTRACT}

In the process of educational inclusion, various ways have been tried to take advantage of the conditions in which basic education takes place, which allows students with disabilities to learn with quality. It is the product of a non-experimental descriptive propositional study, the sample being 183 teachers. It was found that teachers from regular institutions consider the use of basic techniques for the social development of the included child to be deficient, little use of multiple intelligences and active participation. The teachers of the special center, consider that no importance has been placed on the management and values of inclusive teachers before the educational process, such as planning, identifying new opportunities to achieve heterogeneous groups, social development and solving problems and the use of instruments such as the individual orientation plan and the psychopedagogical evaluation. Given this, methodological strategies were designed based on the theory of complexity and multiple intelligences that demonstrates its effectiveness, both in its theoretical conception and in practice, demonstrating effectiveness in the learning achievement of included students.

\section{Keywords}

Teaching method, mental deficiency, disability, student and methodology.

\section{RESUMO}

No processo de inclusão educacional, várias formas têm sido tentadas para aproveitar as condições em que ocorre a educação básica, o que permite que os alunos com deficiência aprendam com qualidade. É o produto de um estudo proposicional descritivo não experimental, tendo a amostra sido de 183 professores. Constatou-se que os professores de instituições regulares consideram o uso de técnicas básicas para o desenvolvimento social da criança incluída como deficiente, pouca utilização de inteligências múltiplas e participação ativa. Os docentes do centro especial, consideram que nenhuma importância tem sido atribuída à gestão e aos valores dos professores inclusivos antes do processo educativo, como o planeamento, identificação de novas oportunidades de concretização de grupos heterogéneos, desenvolvimento social e resolução de problemas e utilização de instrumentos como o plano de orientação individual e a avaliação psicopedagógica. Diante disso, estratégias metodológicas foram elaboradas com base na teoria da complexidade e das inteligências múltiplas que demonstra sua eficácia, tanto em sua concepção teórica quanto na prática, demonstrando eficácia no desempenho de aprendizagem dos alunos incluídos.

\section{Palavras chave}

Método de ensino, deficiência mental, deficiência, aluno e metodologia. 


\section{Introducción}

La educación peruana hace frente a situaciones de exclusión, marginación, rechazo e incluso apatía ante los estudiantes con discapacidad en los diversos niveles educativos, puesto que, a pesar que desde hace tiempo se ha generado un incremento de la conciencia social de la población aun podemos encontrar diferencias, que han llevado a la exclusión.(Ramírez Valbuena, 2017). En ese sentido, la educación inclusiva ya no es sólo alternativa, sino un compromiso a garantizar en todos los niveles y a todos los estudiantes en general, y en consecuencia, encaminar todos los esfuerzos y necesarios, para el fortalecimiento de las escuelas regulares, a fin de hacer de ellas espacios de valoración de la diversidad.(Red de Educación Inclusiva, 2019).

Las escuelas regulares con orientación inclusiva representan el medio más eficaz para combatir las actitudes discriminatorias, crear comunidades receptivas, construir una sociedad integradora y lograr la educación para todos.(Ministerio de Educación, 2012), esto sumada a otros factores necesarios para que un individuo disfrute de una vida segura y productiva como un ser totalmente integrado en la sociedad, independientemente de cualquier condición. (Figueroa Ángel et al., 2017) Sin embargo, existen muchas dudas respecto al desarrollo de prácticas adecuadas que constituyan la educación inclusiva ideal, en aulas inclusivas, dando una respuesta de calidad y valorando el proceso educativo como un proyecto en comunidad donde la participación de todos sea indispensable para generar sinergias en una la sociedad heterogénea.(Tregón Martín, 2017).

El Ministerio de Educación, de acuerdo a la Constitución Política del Perú, la Ley $\mathrm{N}^{\mathrm{o}}$ 28044 y la Ley Orgánica del Ministerio de Educación, a través de la Dirección General de Educación Básica Especial, órgano responsable de la atención de las personas con discapacidad, asume la responsabilidad de proponer los cambios que el sistema educativo requiere para lograr una educación de calidad con equidad para estas personas, en igualdad de condiciones y oportunidades, con la participación de la familia y comunidad.(Ministerio de Educación, 2012). Ante esto, las instituciones educativas generales suelen argumentar que las/os niñas/os con discapacidad no podrán cumplir con las exigencias de la escuela, que no hay vacantes, que se ha cubierto el "cupo por discapacidad", que sus instalaciones no son accesibles o que no cuentan con personal idóneo para llevar adelante el proceso de inclusión. A su vez, es frecuente que condicionen su matrícula a la disponibilidad de personal que cumpla la función de apoyo, 
a los certificados psicológicos, al pago de sumas adicionales.(Red de Educación Inclusiva, 2019).

En el Perú existen documentos legislativos que respaldan los derechos de las personas con discapacidad y que en los últimos años se han percibido mejoras en el acceso educativo. Sin embargo, a menudo las normas no se cumplen, haciendo notar la escasez de estudios sobre las oportunidades de aprendizaje y los resultados de los estudiantes con discapacidad.(Cueto et al., 2018).

A nivel mundial 93 millones de niñas y niños menores de 14 años viven con discapacidad moderada o severa, y según estadísticas en los países con bajo nivel de desarrollo, la posibilidad de escolarización es escasa.(Castillo Morales, 2017). En el sistema educativo peruano, considerando que los CEBEs atienden a una población aproximada de 20,899, podríamos inferir que la brecha existente en la atención a la población con discapacidad severa y multidiscapacidad es muy alta. Por tanto, el desafío mayor está dirigido al acceso, permanencia y atención de calidad a los estudiantes con discapacidad severa $\mathrm{y}$ multidiscapacidad.(Ministerio de Educación, 2012). Por otro lado, la preocupación por incrementar la calidad de la enseñanza se acentúa, debido a que está condicionada a logros académicos y la aplicación de estrategias adecuadas en el trabajo docente. En ese sentido, el trabajo del director y de los docentes en los centros educativos adquiere mayor impulso; y la preocupación por crear estrategias adecuadas en el proceso de aprendizaje es urgente.(MINEDU, 2014). Al respecto, (Kourkoutas, 2011) señala que "las escuelas son importantes contextos sociales de carácter académico en el que niños y niñas, familias, educadores y miembros de la comunidad tienen la oportunidad de interactuar, intercambiar opiniones e ideas, aprender, enseñar y crecer". De manera significativa, los niños desfavorecidos tienden a ser los que menos acceso tienen a una educación y atención a una educación de calidad, a pesar de que son ellos los que más se beneficiarían de una intervención temprana. (Ainscow, 2019).

El principal desafío lo localizamos en cambiar la mirada del docente y del resto del personal hacia el alumnado, y creer que el estudiante con diversidad funcional puede aprender, puede participar, y no solo «estar». Además de creer en la inclusión, se necesita estrategias metodologías activas que son las que nos van a permitir conseguir esos pilares básicos de la inclusión: presencia, participación y aprendizaje.(Galván, 2019). Estos cambios que se requieren a nivel de la escuela y la familia también deben alcanzar a la sociedad, por ello, exigen el compromiso de todos para que se rompan las barreras de la desinformación, los estereotipos, etc. y se permita orientar la vida cotidiana a nuevas 
prácticas donde la inclusión sea algo natural. (MINEDU, 2014). Esto implica que la educación inclusiva sea entendida como un principio más amplio que apoya y atiende la diversidad de todos los alumnos.

(Ainscow, 2019).

Si bien es cierto, que la inclusión varía de acuerdo con circunstancias históricas; tiene en común el propósito de mejorar cada día la sociedad,.(Ramírez Valbuena, 2017). Al mismo tiempo, los principios básicos de la educación inclusiva consideran la comunidad como un medio para alcanzar la equidad e inclusión de los alumnos tanto dentro como fuera del centro educativo.(Tregón Martín, 2017).

Todos estos elementos, le dan una complejidad social que involucra a las familias, en el proceso educativo de sus hijos o hijas, favoreciendo el diálogo sobre aspectos relevantes de la vida escolar de las personas con discapacidad (como los logros académicos, dificultades de aprendizaje, interacción con sus compañeros, entre otros), a los docentes y tutores con las familias, para promover la colaboración en las actividades organizadas por la institución educativa.(Ministerio de Educación, 2013), pero también con otras instancias de la sociedad en general.

Siendo el pensamiento complejo; un pensamiento conector y multidimensional, su finalidad es articular fragmentos disciplinarios antagónicos fracturados por el pensamiento simplificador.(Peñaranda Pando, 2016), por ello, la importancia de concebir estrategias desde el pensamiento complejo y las inteligencias múltiples. En este marco, el presente trabajo tiene como propósito, elaborar una estrategia posible para superar una de sus más fuertes limitaciones: la carencia de una metodología empírica que permita un mejor aprendizaje de los estudiantes con discapacidad incluidos en aulas de Educación Básica en Perú.

La complejidad reside en el carácter recursivo del proceso a través del cual el pensamiento constituye la realidad al tiempo que es constituido por ésta. Lo que llamamos realidad es una estructuración histórica de ese proceso recursivo. La reorganización de un sistema de pensamiento implica, de modo concomitante, transformaciones profundas en la organización de la realidad. (Rodríguez Zoya, 2017). Por ende, el pensamiento abstracto y la conciencia del ser humano se reflejan subjetivamente en estudiantes, pero con un toque de objetividad, el mundo que nos rodea, la naturaleza y la vida social. (Yanes Guzmán, 2016). 
La complejidad como método, puede pensarse no como un camino absolutamente necesario hacia la verdad, sino meramente como una herramienta que vale la pena analizar en cuanto a la potencialidad que entraña para llegar a adquirir conciencia de conocimiento, a adquirir conciencia del ocultamiento de los fenómenos bajo la especie de ruidos informacionales que impiden conocer a los objetos de estudio de manera plena.(Peñaranda Pando, 2016). En dicho método, el análisis del pensamiento complejo no se trata de suprimir las disciplinas, sino por el contrario, se trata de hacerlas aún más útiles, coordinándolas e integrándolas, lo cual implica una educación mental y una estructura de pensamiento capaz de afrontar la complejidad, que es el mejor antídoto contra los pensamientos únicos (Juárez \& Comboni Salinas, 2012) no obstante, la complejidad no es algo nuevo. Todo fenómeno, por más sencillo que parezca, encierra múltiples partes y está siendo influenciando por aspectos varios del entorno llevándolo a la creación de conocimiento.(Chacón Prado, 2015) y una práctica compleja del pensamiento implica la construcción de un pensamiento capaz de pensarse a sí mismo con el fin de problematizarse, conocerse y autocriticarse. En este sentido, el desarrollo del pensamiento complejo es una estrategia para la construcción de un conocimiento reflexivo. (Rodríguez Zoya, 2017).

Ante estas consideraciones, transformar la educación es una tarea principal, que requiere cambios en la percepción de profesores, padres y sociedad en general. Esto crearía una nueva percepción que nos permitiría entender mejor los problemas críticos que hoy encontramos en la educación especial.(Yanes Guzmán, 2016), pues conocer y comprender son dos cosas diferentes de un mismo asunto. Ambas son producto de la mente humana, aunque primero se conoce la parte pensante de la mente; luego, se comprende que está ligado a la inteligencia humana.(Ruiz Lara \& Torres Soler, 2017).

Desde el enfoque de la complejidad, los individuos son concebidos como sujetos sociales sistémicos en situación de influencia recíproca, capaces de establecer, mediante una variedad de actividades y formas de expresión, determinada red compleja de relaciones e interacciones sociales con el entorno del cual forman parte indivisible. Es decir, los sujetos sociales son individuos de algún modo socializados, heterogéneos, constructores de objetos $\mathrm{y}$, sobre todo, personas dinámicas que reconocen al mismo tiempo la presencia significativa de los otros en cada cosa u objeto material que utilizan.(Alvarez Nieto, 2016). Morin invita a reconocer las múltiples incertidumbres que se encuentran presentes que impiden que el conocimiento sea algo absoluto, certero e inamovible.(Alvarez Nieto, 2016). 
La teoría del conocimiento, y la elaboración de un nuevo paradigma en la intervención del proceso inclusivo, se hace imperativo, a partir de reconocer la complejidad de la realidad educativa y social, esta visión se ve estrechamente relacionada como una red de interrelaciones intrincada y también conflictiva (Juárez \& Comboni Salinas, 2012) en el proceso educativo.

Por esto, se considera que debe darse una transformación en la construcción y transmisión del saber; que este debe ser interdisciplinario y tender a ser transdisciplinar, en busca de que el pensamiento sea más amplio y se adapte a nuevos escenarios permitiendo comprender los distintos fenómenos del entorno; esto se lograría mediante procesos educativos dialógicos que incluyan la complejidad y el tradicional reduccionismo, lógico y racional.(Ruiz Lara \& Torres Soler, 2017).

El principio de complejidad que se funda también en la necesidad de distinguir y de analizar, como el principio de explicación clásico, trata de establecer la comunicación entre lo distinguido y quien distingue, entre el observador y lo observado.(Juárez \& Comboni Salinas, 2012). En este contexto, la teoría de las inteligencias múltiples está dotadas por un amplio perfil de capacidades intelectuales, y que cada persona tiene algunas de estas capacidades mas desarrolladas que otras. Esto tiene su repercusión en el ámbito de la educación, ya que significa que si lo que perseguimos es una enseñanza que prime los valores de justicia e igualdad, no podemos enseñar de manera uniforme, paradójicamente a todos los alumnos.(Méndez Valcárcel \& Del Teso Martín, 2017).

La teoría de las inteligencias múltiples propone un nuevo enfoque para el estudio y, supone un modo global para afrontar las diferencias personales y comprender las capacidades e intereses individuales. (Lerma Anguix, 2019). Cada una de las inteligencias se codifica en un sistema simbólico que puede ser diferente, ése sistema es habitualmente un producto cultural, y facilita la codificación, el almacenamiento y la organización de los tipos importantes de información.(Athanassopoulos et al., 2017), implica la habilidad necesaria para resolver problemas o para elaborar productos que son de importancia en un contexto cultural o en una comunidad determinada.

Las inteligencias son potenciales que se realizan o no se realizan, dependiendo del contexto cultural en que se hallan. Las inteligencias son siempre una interacción entre las tendencias biológicas y las oportunidades de aprendizaje que existen en una cultura.(Bartolomé Pina, 2017). Por lo tanto, si cada uno tenemos un perfil de inteligencia distinto y tenemos más dominadas unas inteligencias que otras, aprendemos de maneras distintas y controlamos unas habilidades concretas, a diferencia del resto de individuos. 
En este sentido, resulta obvio mencionar la atención a la diversidad, desde los parámetros básicos de personalización del aprendizaje y desarrollo individualizado. (Lerma Anguix, 2019).

Según la teoría de las inteligencias múltiples, debemos individualizar la educación. El conocimiento de los perfiles individuales resulta fundamental para aprovechar los puntos fuertes del alumnado y compensar las debilidades. Igualmente, resulta importante enseñar los contenidos de maneras distintas para poder llegar al máximo número de niños. Su importancia radica en compartir conocimiento, coeducarnos, entendiéndose como una educación paralela, no vertical, donde mutuamente se acepta que el conocimiento y la ignorancia pueden ser sinónimos o hermanas gemelas que se hospedan en todos los seres humanos sin discriminar edades. Por eso, estas acciones de enseñanza-aprendizaje, en las que se requieren de dos o más personas, no debería tener roles, pero como existen, es necesario aceptar, que lo único conocido es nuestra ignorancia.(Athanassopoulos et al., 2017).

Hay que tener en cuenta que en una clase se encuentran alumnos con diferente nivel curricular, con diferentes necesidades, capacidades, por lo que el proceso de enseñanzaaprendizaje tiene que adaptarse a esas capacidades e intereses llevar a cabo una correcta inclusión.(González Castellano, 2017). Cada una de las inteligencias tiene la misma importancia, aunque unas pueden estar más desarrolladas que otras, todos podemos alcanzar cotas máximas en esas inteligencias innatas y también podemos obtener un desarrollo adecuado e incluso bueno en otras inteligencias, siempre y cuando encontremos un ambiente que ofrezca las condiciones necesarias para ello. (Gutiérrez Delgado, 2017).

Por consiguiente, cada una de las inteligencias se puede mostrar de diferente manera dependiendo del sujeto, no todos los sujetos con buen nivel en una de las inteligencias se comportan de igual modo. Pueden desarrollarse diferentes destrezas para una misma inteligencia dependiendo del sujeto.(Athanassopoulos et al., 2017) Desde este punto, emplear en el aula las Inteligencias Múltiples, permite al docente atraer la atención de los alumnos, pues se utilizan materiales motivadores para trabajar todas las inteligencias, potenciando la autoestima, la motivación, ofreciendo un mejor clima en el aula y en la relación entre compañeros. Además, nos permite tener en cuenta los intereses, capacidades e Inteligencias Múltiples de cada uno de los alumnos; de este modo, el proceso de enseñanza-aprendizaje se puede personalizar adaptándose a sus necesidades, ya que podemos identificar aquellas áreas e inteligencias donde se demande más apoyo, 
facilitando la atención a la diversidad en el aula, consiguiendo una integración de todo el alumnado y de esta manera, los alumnos pueden tener un mejor conocimiento de sí mismos y de su estilo de aprendizaje.(González Castellano, 2017).

Dentro de la teoría de las inteligencias múltiples, hay puntos que conviene destacar cada persona tiene cada una de las inteligencias, que funcionan de manera diferente en cada individuo. La mayoría de los individuos pueden desarrollar cada inteligencia hasta un nivel adecuado de competencia, si se recibe el estimulo adecuado. Las distintas inteligencias trabajan juntas de manera compleja, dado que ninguna inteligencia existe por si misma, sino que se producen en contextos culturalmente valorados. Hay distintas formas de ser inteligente dentro de cada categoría, dado que puede destacarse mucho en unas de ellas y no tanto en el resto.

(Martí Quiles, 2017).

\section{Métodos}

\section{Diseño:}

El paradigma socio-crítico adopta la idea de que la teoría crítica es una ciencia social que no es puramente empírica ni sólo interpretativa; sus contribuciones, se originan de estudios comunitarios. Tiene como objetivo promover las transformaciones sociales, dando respuestas a problemas específicos presentes en el seno de las comunidades, pero con la participación de sus miembros. Afirma que algunos de los principios del paradigma son: (a) conocer y comprender la realidad como praxis; (b) unir teoría y práctica, integrando conocimiento, acción y valores; (c) orientar el conocimiento hacia la liberación del ser humano; y (d) proponer la integración de todos los participantes, en procesos de autorreflexión y de toma de decisiones consensuadas, asumiéndolo de manera corresponsable.

Entre las características más importantes del paradigma socio-crítico aplicado al ámbito de la educación se encuentran: (a) la adopción de una visión global y dialéctica de la realidad educativa; (b) la aceptación compartida de una visión del conocimiento, así como de los procesos implicados en su elaboración; y (c) la asunción de una visión particular de la teoría del conocimiento y de sus relaciones con la realidad y con la práctica.

\section{Participantes}

La población estuvo constituida por 183 distribuidos en docentes del equipo SAANEE, docentes de aula inclusiva y docentes en general de las escuelas regulares. 


\section{Instrumentos}

Se hizo uso de 03 diferentes instrumentos, dirigidos a cada fuente de información:

Encuesta de uso de estrategias de intervención a los docentes del CEBE

Encuesta de estrategias de intervención a los docentes del aula inclusiva

Cuestionario a docentes en general

El enfoque usado fue el no experimental, el diseño apropiado fue el tipo transversal. Ya que se recolectó los datos en un solo momento o tiempo único, que permitió describir las variables de estudio, analizar su incidencia y desarrollar la propuesta.

\section{Procedimiento}

Se desarrollo en dos fases; inicialmente se hizo la descripción con la ayuda de cuadros obtenidos a través de estadística descriptiva de la percepción de los docentes involucrados en la intervención de los estudiantes con discapacidad. En la segunda fase se planteó la estrategia metodológica que fue validada por criterio de jueces realizada para demostrar la utilidad de la estrategia metodológica innovadora.

\section{Aspectos éticos}

Los participantes firmaron el consentimiento informado, donde se constata que recibieron información suficiente sobre los cuestionarios y el procedimiento. Por otra parte, los participantes manifestaron a los investigadores su disposición y permiso, de manera independiente y voluntaria, para brindar el tratamiento de los datos proporcionados en cada uno en los cuestionarios.

\section{Análisis de datos}

En primer lugar, se describe las características de las estrategias metodológicas que se utilizaron en la intervención de los estudiantes incluidos en aulas regulares, se hizo una distribución porcentual de cada una de las dimensiones de las variables en estudio, después de haber aplicado los tres instrumentos; $\mathrm{y}$, por último, por medio de tablas se sistematizó la información recogida. Los datos obtenidos permitieron darle mayor solidez a la sustentación de la propuesta de intervención.

\section{Resultados}


A continuación, se muestra los resultados de las percepciones de los docentes de las aulas regulares en general, de los docentes inclusivos y de los docentes de educación especial.

Tabla $\mathbf{N}^{\circ} 1$.

Percepción de los docentes de aulas regulares sobre las dimensiones de las estrategias utilizadas, de acuerdo a nivel que presenta

Uso de Incorporació Incorporación

Uso de Estrategias de técnicas para $n$ de técnicas

de técnicas

CATEGORIA inteligencias participación

el desarrollo para resolver Tomade múltiples activa social conflictos Decisiones

\begin{tabular}{lccccc}
\hline Optimo & $20,69 \%$ & $27,59 \%$ & $31,03 \%$ & $31,03 \%$ & $17,24 \%$ \\
Bueno & $27,59 \%$ & $17,24 \%$ & $13,79 \%$ & $20,69 \%$ & $17,24 \%$ \\
& & & & & \\
Regular & $37,93 \%$ & $37,93 \%$ & $31,03 \%$ & $31,03 \%$ & $37,93 \%$ \\
& $13,79 \%$ & $17,24 \%$ & $24,14 \%$ & $17,24 \%$ & \\
Deficiente & & & & & \\
& $100,00 \%$ & $100,00 \%$ & $100,00 \%$ & $100,00 \%$ & $100,00 \%$
\end{tabular}

TOTAL

Fuente: Resultado de encuesta a docentes en general

En la tabla $\mathrm{N}^{\circ} 01$, se muestra que del total de encuestados el $51,72 \%$ consideran que el uso de técnicas básicas para el desarrollo social, presentan una condición deficiente o regular, el 51,72\% de docentes hace uso de estrategias de inteligencias múltiples, pero en forma también regular o deficiente; lo mismo ocurre con las estrategias para la participación activa que muestran un nivel deficiente y regular con 55,17\%. Ante estas condiciones, se hace necesario fortalecer estas tres estrategias metodológicas; debido a que solamente el $34,48 \%$ en la incorporación de técnicas para resolver conflictos y la incorporación de técnicas de toma de decisiones. 
Se observa que, del total de docentes de aulas regulares, el 13,79\% señalan que el uso de técnicas básicas para el desarrollo social del niño incluido es absolutamente deficiente, eso implica la generación de condiciones inadecuadas para un proceso inclusivo óptimo. A su vez. el 17,24\% señalan que el uso de estrategias de inteligencias múltiples en las actividades del niño incluido es absolutamente deficiente. Por su parte, el $24,14 \%$ señalan que el uso de técnicas básicas para estrategias de participación activa del niño incluido es absolutamente deficiente. Debe indicarse que el 17,24\% indican que no se han incorporado técnicas para resolver conflictos del niño incluido son absolutamente deficientes. Finalmente, el $17,24 \%$ indican que no se han incorporado técnicas de toma de decisiones en el proceso inclusivo del niño, generando condiciones inadecuadas para un proceso eficiente.

Tabla $N^{\circ} 2$.

Percepción de los docentes de educación especial acerca del uso de estrategias de intervención

$N^{\circ}$ Estrategias de intervención $\quad$ SI $\quad N O$

$10,00 \%$

Prioriza los recursos y potencialidades humanos para 100,00\% optimizar el proceso aprendizaje.

$20,00 \%$ $100,00 \%$

Existe compromiso de los docentes inclusivos en el proceso inclusivo de los estudiantes con necesidades educativas especiales.

Se elabora un plan estratégico de la escuela con una orientación inclusiva

$4 \quad 0,00 \%$

$100,00 \%$

Se socializa con docentes y padres de familia para hacerlos conocer el plan de orientación individual y evaluación psicopedagógica

$5 \quad 0,00 \%$ Está pendiente del proceso de seguimiento y monitoreo del proceso $100,00 \%$ con docentes y padres de familia

Fuente: Resultados de encuesta a docentes de educación especial

La tabla $\mathrm{N}^{\circ}$ 02, muestra que los docentes del Centro de Educación Básica Especial, perciben que solo se prioriza los recursos y potencialidades humanos para optimizar el 
proceso de aprendizaje y que coinciden que no se ha puesto importancia en el compromiso de los docentes inclusivos en el proceso de inclusión de los estudiantes con necesidades educativas especiales, no se elabora un plan de desarrollo de la escuela (PEI) con orientación inclusiva, no se socializa el proceso con docentes y padres de familia para hacerlos conocer el plan de orientación individual y la evaluación psicopedagógica. Finalmente, no están pendientes del proceso de seguimiento y monitoreo del proceso con docentes y padres de familia.

Tabla $N^{\circ} 3$.

Percepción del docente del aula inclusiva sobre los métodos y estrategias

$N^{\circ}$ Estrategias de intervención docente en educación regular $S I$

1 Elaboran una enseñanza cooperativa $\quad 36,00 \%$

$64,00 \%$

2 Está pendiente de un proceso de aprendizaje cooperativo $48,00 \% \quad 52,00 \%$

$3 \quad 36,00 \%$ $64,00 \%$

Se preocupa por el desarrollo y la resolución de problemas colaborativa de los estudiantes

$4 \quad 24,00 \%$ $76,00 \%$

Posee la capacidad de identificar nuevas oportunidades para conseguir agrupaciones heterogéneas

$5 \quad 36,00 \%$ $64,00 \%$

Se revisa el proceso a través de los logros del plan de orientación individual y la evaluación psicopedagógica

Fuente: Resultados de encuesta a docentes inclusivos de instituciones educativas regulares

En la tabla $\mathrm{N}^{\circ} 03$, podemos observar que, del total de docentes del aula inclusiva, el $76,00 \%$ no poseen la capacidad de identificar nuevas oportunidades para conseguir agrupaciones heterogéneas, el $64,00 \%$ no elaboran una enseñanza cooperativa comunes en la planificación, no se preocupa por el desarrollo y la resolución de problemas 
colaborativa de los estudiantes, no revisan el proceso a través de los logros del plan de orientación individual y la evaluación psicopedagógica.

Podemos señalar entonces que se necesita fortalecer aspectos del proceso inclusivo relacionados con: uso de técnicas básicas para el desarrollo social del niño incluido, uso de estrategias de inteligencias múltiples en las actividades, uso de técnicas básicas para estrategias de participación activa, incorporación de técnicas para resolver conflictos e incorporación de técnicas de toma de decisiones del niño incluido.

\section{Discusión}

Los resultados encontrados describen aspectos fragmentados de una misma realidad, ante esto se plantea una propuesta global desde la teoría de la complejidad, donde no se descarta ninguna condición para el análisis y la intervención diversificada que está planteada en las inteligencias múltiples. Dado que es imprescindible adoptar formas eficaces y duraderas para producir la transformación estructural que nuestro sistema de intervención en educación inclusiva requiere. En tal sentido, resulta fundamental y urgente la adopción de algunos presupuestos como lo plantea la Red de Educación Inclusiva (2019).

Diseñar un plan con medidas concretas, plazos e indicadores para transformar las escuelas especiales en escuelas regulares o en centros de apoyo a la inclusión, con el compromiso y participación de la familia. Esto implica una intervención desde la multiplicidad de formas de pensamiento, sentimiento y cultura. En la educación básica se pretende que la diversidad sea una ventaja en dos sentidos: por un lado, permite aprender a convivir y, por otro, promueve la estimulación cognitiva de los educandos (Secretaría de Educación Pública, 2019). En esta complejidad, la participación de la familia es fundamental para la persona con discapacidad ya que en ella se aprenden los valores, hábitos, costumbres y es el soporte para su desarrollo integral, así como el enlace con el mundo externo, por ello es necesario fortalecer las acciones de asistencia a las familias (Ministerio de Educación, 2013). Reestructurar en ellas el modo de percibir y organizar la experiencia de la realidad. Pero, al mismo tiempo, los fenómenos del mundo de la experiencia que condicionan la estructuración del pensamiento complejo (Rodríguez Zoya, 2017). Las actividades deben estar diseñadas para que todos los niños puedan participar, pueden realizarse con toda la clase o separando en grandes o pequeños grupos. Así se puede trabajar de un modo más individualizado y se puede ayudar por si algún alumno tiene dificultades a la hora de realizarlo (Vega Agapito, 2018). 
Desarrollar mecanismos para garantizar la accesibilidad del entorno escolar, la provisión de apoyos y el uso de estrategias metodológicas apropiadas para que cada estudiante pueda aprender y participar en condiciones de igualdad, en virtud de que, a pesar de la presencia de diversas barreras que dificultan la educación de los estudiantes con discapacidad, existen también elementos que facilitan la inclusión de este colectivo.(Cueto et al., 2018), teniendo en cuenta que la promoción de la inclusión y la equidad no consiste en importar prácticas de cualquier parte, si no que, por el contrario, requiere un análisis de la realidad a fin de identificar y abordar los obstáculos experimentados por algunos estudiantes (Ainscow, 2019). Por otra parte, el derecho a la educación inclusiva es un derecho de todas las personas. Los padres, en este sentido, deben tener el papel que les corresponde en el ejercicio del derecho a la educación inclusiva: garantizar que se hace efectivo el derecho de sus hijos a recibir una educación inclusiva de calidad (Pérez Bueno et al., 2017). Sin embargo, los padres junto con los otros miembros de la familia, también son responsables de brindar a los estudiantes espacios y momentos de integración; no obstante, ellos también pueden obstaculizar las relaciones interpersonales entre los estudiantes al actuar de manera sobreprotectora. (Cueto et al., 2018). Considerando que el estudiante es un ser que se adapta a las condiciones del entorno que lo lleva a pasar los límites, pues posee creatividad que debe desarrollarla a través de diferentes estímulos internos y externos (Ruiz Lara \& Torres Soler, 2017), cabe mencionar que las estrategias incluyentes en la educación regular son un tipo de actividades específicas dirigidas a la diversidad estudiantil, teniendo en cuenta sus diferencias individuales, capacidades y habilidades desarrolladas y por desarrollar, las mismas que deben ser organizadas y manejadas por los docentes de la institución. (Morales Calvache, 2018).

Garantizar que los estudiantes con discapacidad certifiquen sus capacidades y logros al igual que el resto de los estudiantes, teniendo en cuenta la diversidad, sigue siendo el mayor reto al que se enfrentan los centros regulares y sus profesionales (Soriano De Gracia, 2011). Sin embargo, es fundamental puesto que, la interacción entre alumnos con distinto rendimiento académico, ya sea entre compañeros del mismo o distinto nivel, beneficia a todos los estudiantes. De este modo, el nivel de aprendizaje global de la clase se incrementa. (Solla, 2013). A su vez, debemos señalar que en torno a la inclusión en la escuela se han generado dos categorías: una centrada en la importancia de los niños y adolescentes, y otra enfocada a favorecer la necesaria interacción entre escuela y familia, por lo tanto en modo complejo, la inclusión social en contextos educativos debe orientarse 
hacia el reconocimiento, promoción y participación de la multiplicidad, singularidad y diversidad de actores, identidades, culturas y contextos, ya que es la diversidad la que enriquece las experiencias, y por ende, los aprendizajes (Figueroa Ángel et al., 2017). Es por esto que asumimos que el pensamiento complejo y el paradigma de la complejidad inducen a desarrollar estrategias para gestionar conocimiento, investigar, permitir que el individuo se asombre del conocimiento, de las acciones que realiza; sobre lo que descubre y entiende para saber qué es y qué está en su entorno (Ruiz Lara \& Torres Soler, 2017). Caracterizándose por ser algo flexible y se puede adaptar a las necesidades personales de cada alumno, tratando de que trabaje aspectos que le cuestan más, mezclados con aquellos que le producen comodidad, consiguiendo una enseñanza individualizada y permitiendo que cada estudiante llegue a su máximo potencial. A la par se pueden trabajar cada una de las inteligencias de manera individual, pero siempre se combinan unas con otras. (Vega Agapito, 2018). Tanto los menores como sus padres tienen que tener reconocida la plena participación y decisión en todos los procedimientos que afecten a la escolarización. (Pérez Bueno et al., 2017).

Promover la formación en el modelo social de discapacidad, brindándoles herramientas que garanticen una educación inclusiva a las personas con discapacidad dentro del sistema de educación general, para lo cual, la educación especial hoy requiere y acepta la diversidad de estrategias que contribuyan en el desarrollo psicosocial de los individuos y en su quehacer diario. (Castillo Morales, 2017). Este modelo de escuela centrado en el individuo tiene en cuenta no solo los intereses, inquietudes, motivaciones, preferencias y experiencias previas de los alumnos, sino también las virtudes que posee cada uno, y su finalidad no es otra que conseguir una verdadera atención a la diversidad que posibilite sacar partido al potencial de todos ellos. (Nadal Vivas, 2015) y mediante el uso de las inteligencias múltiples, se puede conseguir un desarrollo integral del estudiante y además ayudar a los docentes a conocerlo y así poder solventar todas sus necesidades. Teniendo en cuenta que lo que se busca es conseguir que el estudiante sea consciente de que existen diferentes tipos de inteligencias y que, a través de su estimulación, van a conocer la inteligencia donde pueden tener más o menos habilidad, ya que a través de la combinación de las distintas inteligencias podrán tener un mejor progreso en su aprendizaje. (González Castellano, 2017), la concepción pluralista de la inteligencia, pone de manifiesto que la inteligencia tiene múltiples facetas y que todos somos inteligentes en alguna área. Esta manera de concebir al individuo nos aporta una visión más amplia y completa de los alumnos y nos invita a observarlos de una manera más plural, perspectiva que permite a 
los docentes conocerlos y comprenderles mejor pudiendo ajustar con mayor rigor el proceso de enseñanza-aprendizaje a las características cognitivas y personales de cada uno. Otro aspecto que se ha considerado es la educabilidad de la inteligencia, a partir del cual se evidencia la importancia de la tarea docente a la hora de planificar la intervención educativa. Si lo que queremos es favorecer el desarrollo de las capacidades de todos los alumnos, esta teoría nos aporta herramientas más que suficientes para integrar nuevos enfoques e iniciativas en las aulas (Nadal Vivas, 2015). A su vez es fundamental el trabajo cooperativo familia-institución educativa que implica, que estos dos agentes trabajen juntos, para lograr el mismo fin, que es comprender las necesidades educativas especiales de los estudiantes con discapacidad. El éxito de la sociedad familiainstitución educativa, requiere de una mutua colaboración, apoyo y participación de cada uno de los miembros de la familia y la actitud de los profesionales docentes y no docentes. (Ministerio de Educación, 2013). En ese sentido, la estrategia se debe implementar tomando en cuenta criterios de diversidad, territorialidad, interculturalidad e igualdad de género; y avanzar gradualmente en la construcción de la Nueva Escuela Peruana para todas y todos desde la comunidad, superando de manera progresiva la dispersión y precarización que se ha producido por la segmentación del sistema en modalidades y subsistemas para atender a los grupos de mayor pobreza y vulnerabilidad.

Capacitar a docentes y directivos para diseñar, implementar y evaluar acciones relacionadas con la educación inclusiva, asegurando que tengan el liderazgo y las herramientas necesarias para impulsar cambios estructurales. Es imprescindible resignificar la escuela, lo mismo que el rol del docente. Es preciso y fundamental recorrer nuevamente la trama organizativa y pedagógica que sostiene la estructura de las instituciones educativas y que impulsa el desarrollo de las prácticas escolares.(Escobar \& Escobar, 2016). Cambiar las estrategias, es decir el manejo de las formas de abordaje, medios y materiales, que nos orientan, descubren, exploran y aprovechan oportunidades, facilitando el acceso de los estudiantes con necesidades educativas especiales, a las experiencias de aprendizaje que se presentan en el aula. (MINEDU, 2014), producirá un impacto en los estudiantes. Las reacciones serán tan variadas y únicas. Y es a partir de esa respuesta que la educación llevará procesos de reestructuración, transformación y cambio. (Chacón Prado, 2015), integrando el conocimiento pero al mismo tiempo, manteniendo su independencia (Escobar \& Escobar, 2016) Ante esto, la agenda 2030 para el Desarrollo Sostenible, en el objetivo 4 vinculado a educación, propone precisamente la necesidad de eliminar las disparidades en la educación y asegurar el 
acceso igualitario a todos los niveles de la enseñanza y la formación profesional para las personas en situación de vulnerabilidad, incluidas las personas con discapacidad.(OEA (Organización de Estados Americanos), 2017). La reorganización de un sistema de pensamiento procede por la invención de nuevos cuestionamientos que permitieron elaborar los problemas de manera diferente. (Rodríguez Zoya, 2017).

Asegurar que las personas con discapacidad participen y sean escuchadas de manera efectiva en los procesos de toma de decisiones relacionadas con sus derechos, empero habría que destacar que la sola presencia de los alumnos con necesidades educativas específicas, por causa de sus condiciones personales, sociales o culturales, no nos libra del riesgo de que la deseada convivencia, amistad, empatía y solidaridad entre los alumnos con situaciones vitales muy diferentes no sea satisfactoria. Hace falta atención y cuidado permanente de los profesores y de las familias, de las instituciones educativas y sociales, para que las experiencias educativas dentro y fuera de la escuela sean positivas.(Marchesi et al., 2014). Así pues, no debemos perder de vista el principio de la teoría de Gardner, que se basa en la idea de que todos y cada uno de nosotros somos buenos en algún aspecto, todos tenemos talentos y habilidades.(Nadal Vivas, 2015), y que para la atención del estudiante con discapacidad, lo esencial y prioritario es establecer una adecuada relación afectiva con él, para lo cual hay que conocerlo en todas sus manifestaciones, en diferentes ámbitos y en diferentes momentos.(MINEDU, 2014). De alguna forma, el ampliar tanto la concepción de inteligencia no tiene otro objetivo que orientar a la función educadora hacia el desarrollo de personas más inteligentes en la vida real, por diversos caminos. De ahí el esfuerzo por incluir continuamente estrategias de aprendizaje para ampliar y enriquecer las dimensiones propuestas. Se entiende por tanto que este planteamiento de la inteligencia es un proceso abierto y un proceso que se extiende a lo largo de toda nuestra vida (Bartolomé Pina, 2017). Por lo tanto, para favorecer los procesos de inclusión es necesario promover niveles de relación entre estudiantes y entre estos y los demás miembros de la escuela, a través de estrategias como la participación en equipos deportivos, culturales, académicos y extraescolares, que permitan reconocerse como sujetos multidimensionales, así como escenarios de expresión de sentimientos y expectativas en un ambiente de acogida y confianza (Figueroa Ángel et al., 2017), construyendo nuevos conocimientos en los estudiantes, fomentando la participación, capacidad y habilidad, mediante actividades lúdicas de integración y motivación (Morales Calvache, 2018). 
La cuestión clave que se plantea es trabajar estrategias metodológicas que repercutan en los diversos espacios de interacción del niño con discapacidad, dentro del aula teniendo en cuenta las diferencias y en el entorno social para responder de modo más competitivo. Tres aspectos generales pueden señalarse en esta propuesta: Lo que es bueno para los alumnos con necesidades educativas especiales es bueno para todos los alumnos. Los problemas de conducta, sociales o emocionales son los que crean más dificultades en los centros educativos regulares. En las clases de los centros educativos regulares, lo habitual es que exista un grupo numeroso de alumnos y alumnas con edad similar, pero con necesidades de aprendizaje y características socioculturales muy diversas (Solla, 2013). En cuanto a los principios que orientan estas estrategia son los siguientes: a) evaluación flexible, sin etiqueta de los estudiantes en riesgo, centrada en las capacidades y no en la discapacidad; b) enfoque personalizable de acuerdo a las necesidades, intereses y habilidades de los estudiantes, c) fortalecimiento de las capacidades socio-emocionales mediante la introducción en las actividades desde el punto de vista psicológico, d) cooperar con la familia y las relaciones de apoyo (Kourkoutas, 2011).

\section{Conclusiones}

Se encontró que el proceso inclusivo presenta dificultades en la coherencia de las actividades que responden a las necesidades inclusivas, el manejo de la complejidad en las estrategias basadas en las inteligencias múltiples y en el uso de metodología participativa no ha sido asumido aun en el proceso de aprendizaje de los estudiantes con discapacidad incluidos en las instituciones educativas de la educación básica peruana.

El Diseño de Estrategias metodológicas de intervención basada en la Teoría de Morin y las inteligencias múltiples de Gardner para mejorar el proceso de enseñanza aprendizaje en los estudiantes con discapacidad incluidos en aulas de las instituciones educativas regulares, brinda aspectos que facilitan el proceso inclusivo y el proceso de aprendizaje generando condiciones que facilitan el trabajo al docente en el aula, la cooperación entre el docente del centro especial e inclusivo y el logro que se desea alcanzar para mejorar las condiciones de vida de una persona con discapacidad.

Las estrategias metodológicas con el uso de las inteligencias múltiples y la teoría de la complejidad de Morin muestran argumentos que permite mejorar el proceso inclusivo, haciéndolo más efectivo y asumiendo una mirada global del proceso educativo. 


\section{Referencias}

Ainscow, M. (2019). 25 años después de la Declaración de Salamanca de la UNESCO Crear sistemas educativos inclusivos y equitativos. Documento de Discusión Preparado Para El Foro Internacional Sobre Inclusión y Equidad., 11-13.

Alvarez Nieto, G. (2016). El conocimiento del conocimiento: la obra de Edgar Morin y la problemática de la educación mexicana. IE Revista de Investigación Educativa de La REDIECH, 7(13), 6-20.

Athanassopoulos, N., López-Fernández, V., \& López Fernández, V. (2017). Inteligencias múltiples y aprendizaje: Un enfoque comparativo en alumnos de conservatorio. ReiDoCrea: Revista Electrónica de Investigación y Docencia Creativa, 6, 50-63.

Bartolomé Pina, M. (2017). Diversidad educativa ¿Un potencial desconocido? Revista de Investigacion Educativa, 35(1), 15-33. https://doi.org/10.6018/rie.35.1.275031

Castillo Morales, M. M. (2017). Proyecto de inclusión social para niños con discapacidad en colegios privados de educación básica primaria en Facatativa. Corporación Universitaria Minuto de Dios, 53(9), 1689-1699. https://doi.org/10.1017/CBO9781107415324.004

Chacón Prado, M. de J. (2015). Congruencia del pensamiento complejo de Edgar Morín en la metodología de la educación a distancia. Revista Espiga, 14(30), 75-82. https://doi.org/10.22458/re.v14i30.933

Cueto, S., Rojas, V., Dammert, M., \& Felipe, C. (2018). Cobertura, oportunidades y percepciones sobre la educación inclusiva en el Perú. In Grade: Grupo de Análisis para el Desarrollo (Vol. 1, Issue 9). https://doi.org/10.1017/CBO9781107415324.004

Escobar, R. A., \& Escobar, R. A. (2016). La relación entre el pensamiento complejo, la educación y la pedagogía. Reflexión, 46(1), 88-99.

Figueroa Ángel, M. X., Gutiérrez de Piñeres Botero, C., \& Velázquez León, J. (2017). Estrategias de inclusión en contextos escolares. Diversitas, 13(1), 13. https://doi.org/10.15332/s1794-9998.2017.0001.01

Galván, E. (2019). La educación inclusiva, una inversión de futuro. Voces: Plena Inclusión, 22.

González Castellano, N. (2017). Multiple Intelligences and Learning Difficulties. MLS Educational Research, 1(1), 19-38. https://doi.org/10.29314/mlser.v1i1.26 
Gutiérrez Delgado, I. (2017). La teoría de las inteligencias multiples de Down. Cuando el talento se transforma en inteligencia. Investigación En Discapacidad, 6, 2017-2122. www.medigraphic.org.mx

Juárez, J. M., \& Comboni Salinas, S. (2012). Epistemología del pensamiento complejo. Reencuentro, 65, 38-51.

Kourkoutas, E. (2011). Reflexiones sobre la inclusión y los modelos de intervención en la escuela para el alumnado con necesidades educativas especiales. Campo Abierto:

Revista de Educación, 30(2), 127-138.

Lerma Anguix, I. (2019). Las inteligencias múltiples en el aula. Campus Educación. Revista Digital Docente, IV(14), 50.

Marchesi, A., Blanco, R., \& Hernández, L. (2014). Avances y desafios de la educación inclusiva en Iberoamérica Organización de estados americanos para la Educación la Ciencia y la Cultura. 175.

Martí Quiles, L. (2017). Aplicación de la teoría de las inteligencias múltiples y sus efectos sobre el rendimiento y la motivación en alumnos de 4 y 5 de educación primaria. Universidad de Alicante, 279.

Méndez Valcárcel, A., \& Del Teso Martín, E. (2017). La teoría de las inteligencias múltiples aplicada a la enseñanza del inglés como lengua extranjera. Universidad de Oviedo, 51.

MINEDU. (2014). Guía para orientar la intervención de los Servicios de Apoyo y Asesoramiento para la Atención de las Necesidades Educativas Especiales SAANEE.

Dirección General de Educación Básica Especial,
$1, \quad 110$.

http://www.minedu.gob.pe/minedu/archivos/a/002/05-bibliografia-para-ebe/7guiaparaorientar-la-intervencion-de-los-saanee.pdf

Ministerio de Educación. (2012). Educación básica especial y educación inclusiva. Balance y perspectivas. Ministerio de Educación, 1-106.

http://www.minedu.gob.pe/minedu/archivos/a/002/05-bibliografia-paraebe/9educacionbasica-especial-y-educacion-inclusiva-balance-y-perspectivas.pdf Ministerio de Educación. (2013). Guía del Docente para orientar a las familias. Dirección General de Educación Básica Especial, 48. https://doi.org/10.1017/CBO9781107415324.004 Nadal Vivas, B. (2015). Revista de Educación Inclusiva. Revista de Educación Inclusiva, 8(3), 121-136. https://dialnet.unirioja.es/servlet/articulo?codigo $=5446538$ 
Peñaranda Pando, J. P. (2016). Pensamiento Complejo De Edgar Morin : Nueva Visión Del Conocimiento. Reflexión, 11, 129-137.

Ramírez Valbuena, W. Á. (2017). La inclusión: una historia de exclusión en el proceso de enseñanza aprendizaje. Cuadernos de Lingüística Hispánica, 30, 211-230. https://doi.org/10.19053/0121053x.n30.0.6195

Red de Educación Inclusiva. (2019). Educación inclusiva y de calidad. Red Regional Por La Educaciión Inclusiva, 1, 12. https://elperuano.pe/noticia-educacion-inclusivaycalidad74093.aspx

Rodríguez Zoya, L. G. (2017). Problematización de la complejidad de los sistemas de pensamiento: un modelo epistemológico para la investigación empírica de los paradigmas. Revista Latinoamericana de Metodología de Las Ciencias Sociales, 7(2), 025. https://doi.org/10.24215/18537863e025

Ruiz Lara, B. C., \& Torres Soler, L. C. (2017). Pensamiento complejo: transformación del aprendizaje. Comunicación, Cultura y Política, 7, 213-240. https://doi.org/10.21158/21451494.n0.2016.1770

Solla, C. (2013). Guía de buenas prácticas en educación inclusiva. In save the children España. www.observatoriodelainfancia.es/oia/esp/documentos_ficha.aspx?id=4114 Tregón Martín, N. (2017). Orientación educativa para la diversidad funcional visual en Nicaragua. Diseño y evaluación de un programa de formación para el profesorado. Universitat Jaume, 495. https://doi.org/10.6035/14003.2017.214069

Yanes Guzmán, J. R. (2016). Pensamiento complejo abstracto en el aula. Sophía, 2(21), 121.

https://doi.org/10.17163/soph.n21.2016.05 